\title{
The first confirmed gravitational wave detection in LIGO's second observational run
}

\author{
Jin $\mathrm{Li}^{1}$, and XiLong $\mathrm{Fan}^{2 *}$ \\ ${ }^{1}$ College of Physics, Chongqing University, Chongqing 401331, China; \\ ${ }^{2}$ School of Physics and Electronics Information, Hubei University of Education, Wuhan 430205, China
}

Received August 2, 2017; accepted August 22, 2017; published online October 27, 2017

Citation: J. Li, and X. L. Fan, The first confirmed gravitational wave detection in LIGO's second observational run, Sci. China-Phys. Mech. Astron. 60, 120431 (2017), https://doi.org/10.1007/s11433-017-9094-8

In this article, we describe the results concerning the third coincident signal GW170104 from the coalescence of binary black holes (BBHs) during the second observation run $(\mathrm{O} 2)$. The result was obtained from the LIGO Scientific Collaboration and the Virgo Collaboration. Following the first and second gravitational waves (GWs) detections in the first observation run $(\mathrm{O} 1)$ [1], recently LIGO has observed a third coincident signal GW170104 from the coalescence of BBHs during the second observation run $(\mathrm{O} 2)$ [2]. The observation was made on January 4, 2017 at 10:11:58.6 UTC through the Hanford and Livingston detectors.

The search procedures for this detection were similar to the previous events. With improved data quality and detector sensitivity, the LIGO and Virgo Collaborations found no evidence of instrumental or environmental noise that may have contributed to the GW170104 signal. Based on the matched filter method, the event was detected by the waveform-based PyCBC pipeline [3,4] with a network signal-to-noise ratio (SNR) of 13 and a false alarm rate of less than 1 in 70000 years; the event was later independently identified by other pipelines.

From a coherent Bayesian analysis of the data in both detectors $[5,6]$, the values of the source parameters were recovered by the collaborations with $90 \%$ credible intervals. The coalescence of BBHs was located at a luminosity distance, $D_{\mathrm{L}}$, or about 3 billion light years, which is the farthest dis-

*Corresponding author (email: fanxilong@outlook.com) tance recorded so far. Therefore the successful detection of such a weak signal at a greater distance has given more confidence. The total mass of the source is $\sim 50 M_{\odot}$, which is in the mass gap of the GW151226 source $\left(\sim 21 M_{\odot}\right)$ and the GW150914 source $\left(\sim 62 M_{\odot}\right)$. In theory, the formation of such BBHs systems is suggested in a sub-solar metallicity environment [7]. As a subdominant parameter of the BBHs, their effective inspiral spin is inferred, which implies that black holes might spin in any direction [2]. This is the first time that the LIGO and Virgo Collaborations have obtained experimental evidence to show that the spins of BBHs may be antialigned (not excluding zero spins), but the probability seems very low. This research is meaningful in distinguishing the theoretical models for BBHs' formation.

Combined with previous events, it provides more confidence for the result of the general relativity (GR) testing. From the modified dispersion test [8-16] and waveform parametrized test [17-25], additional tight constraints on the deviations from GR were obtained. Until now, no significant departure from GR has been found.

Searches: As with the searches from the previous detections, the analysis search for the source of GW170104, which is also included online and offline searches in each pipline. First, in the online searches, GW170104 was identified by inspection of low-latency triggers in L1 due to the temporary incorrect condition of the Hanford detector in the low-latency system $[4,26]$. The online low-latency search can provide alerts when a GW signal is propagated to the detectors within 
minutes. After collecting the data from triggers over several days and weeks, the quantity of data and the detector calibration were refined.

Based on the matched-filter method, many pipelines have waveform templates with building-discrete banks to cover the search parameter space, correlating the data with each waveform in bank to produce a list of triggers, and executing coincidence tests to find candidate events from the triggers. There are several different pipelines, such as PyCBC pipeline [3, 4], cWB pipeline [27-29], oLIB pipline [30], BayesWave pipeline [31, 32], GstLAL pipeline (https://wiki.ligo.org/DASWG/GstLAL) and so on. In this study, the PyCBC pipeline will be briefly introduced in the subsection of confidence.

In this search, the parameter space was composed of the $\mathrm{BBH}$ systems with a total mass between $2 M_{\odot}$ and (100-500) $M_{\odot}$ and between both the component spins $\epsilon$ $[-0.99,0.99]$ [33]. Determination of the upper boundary of the total mass relied on the detector's sensitivity. Currently the maximum mass boundary in $\mathrm{O} 1$ is set as $100 M_{\odot}$ and then is increased to $500 M_{\odot}$ in $\mathrm{O} 2$. With these conditions, the construction of waveform templates can be derived from two existing algorithms: a geometric-based aligned-spin algorithm $[34,35]$ and a "stochastic" algorithm [33,36,37] .

The correlating step is done by multiplying the template and the data weighted by the power spectrum in the frequency domain. Conduction an inverse Fourier transform of these data will yield the time series of the matched filtering as $z(t)$. On the other hand, for a given waveform template the equivalent filter output of the SNR threshold can be calculated as $z^{*}=\rho^{*} \sigma_{\mathrm{T}} / 2 d y n$, where $\rho^{*}$ is the user-defined threshold on the SNR, $\sigma_{\mathrm{T}}$ is the variance of the template and $d y n$ is dynamic range factor. By comparing the $z(t)$ to $z^{*}$, the triggers can be obtained when $z(t)>z^{*}$.

In the coincidence test, the triggers were observed within a given time window of $\delta t$ between each other for the Hanford detector, and $\delta t+10 \mathrm{~ms}$ at the Hanford-Livingston pairs, which deemed them to be the candidate events. The $10 \mathrm{~ms}$ interval is the travel time for extreme case when the GWs are emitted from a distant source along the line connecting the $\mathrm{H} 1$ and L1. The value of $\delta t$ that describes the uncertainty in the arrival time of weak signals was tuned by injections and timeslides that can be fixed by evaluating how wide the window would be to recover as many added injections as possible to the data while keeping the rate of accidental coincidences low. Based in the current quality of the data and the system sensitivity, a reasonably safe value for this search was found to be $\delta t=5 \mathrm{~ms}$ [38].

Confidence: This search used an improved detection statistic, which was also used to unambiguously identify GW150914 and GW151226 [3]. By reducing the false alarm rate of the search across the entire target signal space (the false alarm rate of $\mathrm{PyCBC}$ is a function of the re-weighted SNR used by the search (Figure 1)) [3], the sensitivity of the BBHs mergers search is improved. In PyCBC analysis, a population of sources with an isotropic distribution in sky location, orientation, and spin angle are simulated and inserted into the data from O1. GW170104 was detected with a network matched-filter SNR of 13 [2]. Using simulated signals, additional improvements in the $\mathrm{BBHs}$ detection rate of advanced LIGO's O1 is considered to have increased by $30 \%$. Currently the PyCBC analysis is also used in binary merger searches in the Advanced LIGO's O2 [4, 38, 39]. Significant candidates can be determined by comparing their detection statistic values to an estimate of the background noise. For GW170104, the false alarm rate was less than 1 per 70000 years [2].

Waveform reconstruction: As a central challenge in Gravitational Wave Astronomy, the process of reconstructing the GW waveform from non-stationary and non-Gaussian noise has been widely studied [40-42]. At present, a morphology-independent wavelet analysis, which is termed Bayes Wave algorithm [31, 43] derived from a concatenation of Bayesian statistics and Morlet-Gabor wavelet frame, works for GW170104 detection. The Bayes Wave algorithm models the detected data $s$ that is composed of GW signals $\boldsymbol{h}$, instrument noise (i.e., glitches) $\boldsymbol{g}$ and Gaussian noise $\boldsymbol{n}: \boldsymbol{s}=\boldsymbol{R} * \boldsymbol{h}+\boldsymbol{g}+\boldsymbol{n}$, where $\boldsymbol{R}$ is a time-delay operator describing the detector's response to the GW strain $\boldsymbol{h}$ [31]. The component of target GW signal wavelets in frequency domain can be derived as:

$$
\begin{aligned}
(\boldsymbol{R} * \boldsymbol{h})_{i}(f)= & \left(F_{i}^{+}(\theta, \phi, \psi) h_{+}(f)+F_{i}^{\times}(\theta, \phi, \psi) h_{\times}(f)\right) \\
& \times \mathrm{e}^{2 \pi \mathrm{i} f \Delta t_{i}(\theta, \phi)},
\end{aligned}
$$

where

$\boldsymbol{h}_{+}=\sum_{j=0}^{N} \Psi\left(f ; A_{j}, f_{0 j}, Q_{j}, t_{0 j}, \phi_{0 j}\right) ; h_{\times}=\epsilon h_{+} \mathrm{e}^{\mathrm{i} \pi / 2}$,

and $\Psi\left(f ; A_{j}, f_{0 j}, Q_{j}, t_{0 j}, \phi_{0 j}\right)$ depends on the gravitational waveforms in template, where $A_{j}, f_{0 j}, Q_{j}, t_{0 j}, \phi_{0 j}$ form the

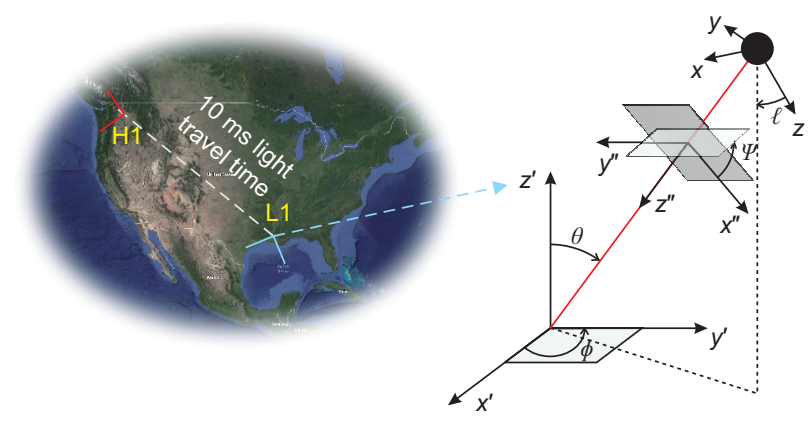

Figure 1 (Color online) Relative position of Hanford and Livingston pairs, illustration of source frame $(x, y, z)$, radiation frame $\left(x^{\prime \prime}, y^{\prime \prime}, z^{\prime \prime}\right)$, detector frame $\left(x^{\prime}, y^{\prime}, z^{\prime}\right)$. 
parameter space of the template. The antenna patterns $F_{i}^{+}(\theta, \phi, \psi)$ and $F_{i}^{\times}(\theta, \phi, \psi)$ will be discussed in the following paragraph. Here the spectrum of the Gaussian component $\boldsymbol{n}$ can be modeled by the Bayes Line algorithm [44,45]. The glitches in the Bayes Wave algorithm $\boldsymbol{g}$ are modeled by a trans-dimensional Reversible Jump Markov Chain Monte Carlo [46] with a variable number of Morlet-Gabor wavelets. The number and parameters of the wavelets would be determined from the data.

The GW170104 signal from the waveform reconstruction has different amplitudes, and it almost has inverse phase at the Hanford and Livingston detectors [2]. Similar phenomenon also appeared in GW150914 [29] and GW151226 [47]. The reason for these phenomena is the detectors' different spatial orientations. In reality, a GW originates from any direction in the sky and its orientation may be such that the detectors can only capture some portion of it. Thus the strain produce at the detector is given by

$h(t)=h_{+}(t) F_{+}(\theta, \phi, \psi)+h_{\times}(t) F_{\times}(\theta, \phi, \psi)$,

where the antenna patterns $F_{+}(\theta, \phi, \psi)$ and $F_{\times}(\theta, \phi, \psi)$ are given by [48]

$F_{+}=-\frac{1}{2}\left(1+\cos ^{2} \theta\right) \cos 2 \phi \cos 2 \psi-\cos \theta \sin 2 \phi \sin 2 \psi$,

$F_{\times}=\frac{1}{2}\left(1+\cos ^{2} \theta\right) \cos 2 \phi \sin 2 \psi-\cos \theta \sin 2 \phi \cos 2 \psi$.

The angles $(\theta, \phi, \psi)$ describing the relative orientation of source and detector are displayed in Figure 1.

Source properties: GW170104 in the frequency band 30$350 \mathrm{~Hz}$ [1] is from the farthest BBHs source observed to date. Through matched filtering pipelines with a network SNR of 13, GW170104 was detected by the Advanced LIGO during its second observation run. The parameters of compact $\mathrm{BBH}$ source can be recovered [5, 6] using LAL Inference [5] software library for Bayesian parameter estimation of compact binary signals. Table 1 shows some source parameters of GW170104 with 90\% credible intervals (Adapted from Table 1 of ref. [2]).

Mass and spin can inferred from the waveform as the most important parameters of black holes. Here, we describe an approximate approach to find the relationship of the waveform varying with mass and spin. The chirp mass $\mathcal{M}=\frac{\left(m_{1} m_{2}\right)^{3 / 5}}{M^{1 / 5}} \simeq \frac{c^{3}}{G}\left(\frac{5}{96} \pi^{-8 / 3} f^{-11 / 3} \dot{f}\right)^{3 / 5}$, plays an important role in the inspiral phase; it can be obtained from the inspiral frequency and its derivative, and the total mass $\boldsymbol{M}$ can inferred from the peak value of the detected strain. Comparing the reconstructed waveforms of the three detections (Figure 1) $[2,29,47]$, the GW signal in the ringdown phases can easily be determined from the final black mass $\boldsymbol{M}$ and spin â. Considering the most lasting mode (i.e., $1=m=2$ ), the central frequency is given as:

$f_{0}=\frac{1}{2 \pi} \frac{c^{3}}{G M}\left(1.5251-1.1568(1-\hat{a})^{0.1292}\right)$.

The corresponding peak strain amplitude mainly relies on the total mass and source luminosity distance $D_{\mathrm{L}}$. It can be expressed as:

$h_{\max }=\frac{G M}{c^{2} \times D_{\mathrm{L}}} \sqrt{\frac{5 \epsilon}{2}} Q^{-1 / 2} F(Q)^{-1 / 2} g(\hat{a})^{-1 / 2}$,

where $Q=0.7+4187(1-\hat{a})^{-0.4990}, F(Q)=1+7 / 24 Q^{2}$, $g(\hat{a})=1.5251-1.1568(1-a)^{0.1292}$.

From Figures 2 and 3, it can be observed that, from the given spin and effective distance, increasing the total mass causes significant decline in the frequency of the ringdown signal, wheras the peak value of the strain increases linearly.

The latest observation also provides some useful information related to the orientations of the black hole spins which evolve with the precession of the inspiral orbital $[49,50]$. Since the spins of black holes have a close relationship to its formation, these measurements can potentially be used to trace the channel of their formation. The effective inspiral spin parameter $\chi_{\text {eff }}$ is defined as $\chi_{\text {eff }}=\left(m_{1} a_{1} \cos \theta_{L S_{1}}+\right.$ $\left.m_{2} a_{2} \cos \theta_{L S_{2}}\right) / M$ [51-58], where $\theta_{L S_{i}}=\cos ^{-1}\left(\hat{\boldsymbol{L}} \cdot \hat{\boldsymbol{S}}_{i}\right)$ is the tilt angle between the spin $S_{i}$ and the orbital angular momentum $\boldsymbol{L}\left(0^{\circ}\right.$ (spin aligned) $\leq \theta_{L S_{i}} \leq 180^{\circ}$ (spin anti-aligned)). In Table 1 this is inferred at a point in the inspiral phase $[2,6]$. From the recovered source parameters, the effective inspiral spin parameter of GW170104 was considered to be $-0.12_{-0.30}^{+0.21}$. Although the orientations of this GW source spin are still undetermined, they can be used to illustrate the possibility of $\chi_{\text {eff }}<0$ [2], which means that total spin might be anti-aligned compared with the overall orbital motion, though it is a slim chance. Such results favor dynamic formation in dense star clusters, where the black holes can spin in any direction relative to their orbital motion [59]. So far, the detections have not found evidence to support another main formation channel, which led to the proposal of isolated binary evolution in galactic fields with aligned spin [60].

Table 1 Source parameters for GW170104 in source frame: median values with 90\% credible intervals (adapted from Table 1 of ref. [2])

\begin{tabular}{|c|c|c|c|c|c|c|c|c|}
\hline $\begin{array}{c}\text { Primary black } \\
\text { hole mass }\end{array}$ & $\begin{array}{c}\text { Secondary black } \\
\text { hole mass }\end{array}$ & Chirp mass & Total mass & Final mass & $\begin{array}{c}\text { Effective } \\
\text { inspiral spin }\end{array}$ & $\begin{array}{c}\text { Final black } \\
\text { hole spin }\end{array}$ & $\begin{array}{l}\text { Luminosity } \\
\text { distance }\end{array}$ & Source redshift \\
\hline $31.2_{-6.0}^{+8.4} M_{\odot}$ & $19.4_{-5.9}^{+5.3} M_{\odot}$ & $21.1_{-2.7}^{+2.4} M_{\odot}$ & $50.7_{-5.0}^{+5.9} M_{\odot}$ & $48.7_{-4.6}^{+5.7} M_{\odot}$ & $-0.12_{-0.30}^{+0.21}$ & $0.64_{-0.20}^{+0.09}$ & $880_{-390}^{+450}$ & $0.18_{-0.07}^{+0.08}$ \\
\hline
\end{tabular}




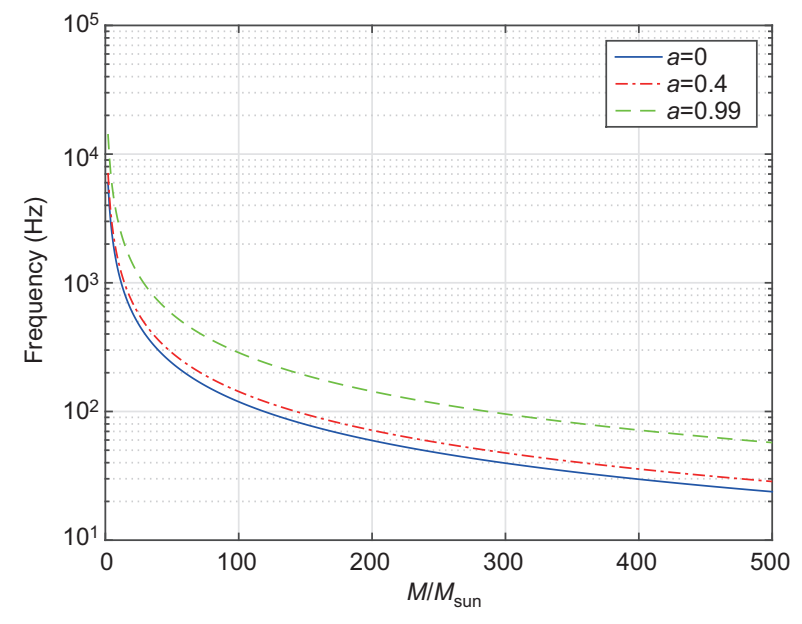

Figure 2 (Color online) Frequency of GW in ringdown phase versus mass for final spin $\hat{a}=0$ (blue line), $\hat{a}=0.4$ (red dash-dot), $\hat{a}=0.99$ (green dashes).

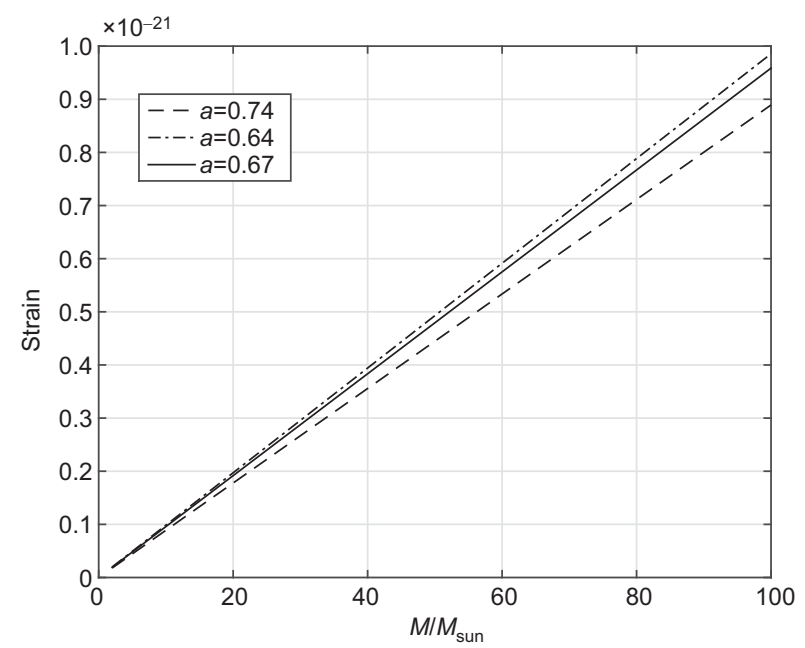

Figure 3 The peak strain amplitude versus mass for the final spin equaling to the three detected BBHs respectively. Here for the lines the source luminosity distance is $613.19 \mathrm{Mpc}$.

Checking the consistency of the observed GW with the predictions in the GR theory is one of the most important objectives of LIGO's detection. Until now, there are three confident detections (GW150914, GW151226 and GW170104). From these, some deviations from GR can be tested, such as dispersion in GW [8-10] and waveform consistency [17-19]. For dispersion testing, the energy of GW radiation is modified by the amplitude of the dispersion $A$ and also by the factor of modified gravity theory $\alpha$ [11-15]. As a result an extra phase shift term appears [8] which can be constrained in the effective-precession waveform [16]. The results show an upper bound of $|A|$ is $\sim 10^{-19}$ (GR corresponds to $A=0$ ) [2]. For waveform consistency verification, null testing was performed, where the deviation coefficients of the phase expansion in their nominal GR values were allowed; a posterior distribution for the difference between measured and GR values was obtained [20-25]. Consequently, none of the tests showed a statistically significant deviation from the GR value [2].

Combining the GW detection with other astronomical observatories will open a new era of multi-messenger astronomy, which aims to combine observations ranging from sensitive detectors to various messenger particles. Since the first (GW150914) and second GW (GW151226) detections, few corresponding joint analysis is in progress, for e.g., Highenergy Neutrino-GW using neutrino observations to help localize the sources and enable the study of their astrophysical progenitors [61-63]; EM-GW using GWs and photons to provide complementary information about the physics of the source and its environment ${ }^{* 11}$.

Conclusions: Advanced LIGO began its second observation run $(\mathrm{O} 2)$ at the end of the November of last year after more than a month of the occurrence of previous $\mathrm{GW}$ event (i.e., GW170104); GW170104 originated from the farthest detected BBH source to date. This indicates that the improved data quality and system sensitivity greatly enhanced the ability of the detection. Additional innovative points of this detection include the following: using of improved data and system sensitivity, extending its observatory size in the universe, finding the probability of anti-aligned spin to filter the formation models of BBHs, further narrowing the deviation from GR. These parameters will help in the development of future detections and its corresponding physical meanings.

The third observation run of the Advanced LIGO will begin in late 2018, and it is hoped that more GWs events will be observed from BBHs as well as from other GW signals (e.g., from Neutron star (NS)-NS). These GW signals, together with their electromagnetic counterpart signals, will lead to opening of a new research field, termed "multi-messenger" astronomy [64-66].

This work was supported by the National Natural Science Foundation of China (Grant Nos. 11673008, and 11205254), the Newton International Fellowship Alumni Follow on Funding, the Fundamental Research Funds for the Central Universities (Grant No. 106112016CDJXY300002), and Chinese State Scholarship Fund. We have presented a summary of the findings from the first aLIGO observational run. Much of the information reviewed here appears in a body of literature, referenced herein, that the LIGO Scientific Collaboration and Virgo Collaboration published throughout 2016. This body of work was the product of our many dedicated colleagues who contributed to the overall understanding of what Nature was telling us with our first detections. We also gratefully acknowledge the support of the United States National Science Foundation (NSF) for the construction and operation of the LIGO Laboratory and Advanced LIGO as well as the Science and Technology Facilities Council (STFC) of the United Kingdom, the MaxPlanck Society (MPS), and the State of Niedersachsen, Germany, for support of the construction of Advanced LIGO and construction and operation of the GEO600 detector. Additional support for Advanced LIGO was provided by the Australian Research Council.

1) *http://moriond.in2p3.fr/grav/2017/transparencies/6_friday/1_morning/1_patricelli.pdf 
1 B. P. Abbott et al. (LIGO Scientific Collaboration, Virgo Collaboration), Phys. Rev. X 6, 041015 (2016), arXiv: 1606.04856.

2 B. P. Abbott, et al. (LIGO Scientific Collaboration), Phys. Rev. Lett. 118, 221101 (2017).

3 A. H. Nitz, T. Dent, T. D. Canton, S. Fairhurst, and D. A. Brown, (2017), arXiv: 1705.01513.

4 S. A. Usman, A. H. Nitz, I. W. Harry, C. M. Biwer, D. A. Brown, M. Cabero, C. D. Capano, T. D. Canton, T. Dent, S. Fairhurst, M. S. Kehl, D. Keppel, B. Krishnan, A. Lenon, A. Lundgren, A. B. Nielsen, L. P. Pekowsky, H. P. Pfeiffer, P. R. Saulson, M. West, and J. L. Willis, Class. Quantum Grav. 33, 215004 (2016), arXiv: 1508.02357.

5 J. Veitch, V. Raymond, B. Farr, W. Farr, P. Graff, S. Vitale, B. Aylott, K. Blackburn, N. Christensen, M. Coughlin, W. Del Pozzo, F. Feroz, J. Gair, C. J. Haster, V. Kalogera, T. Littenberg, I. Mandel, R. O’Shaughnessy, M. Pitkin, C. Rodriguez, C. Röver, T. Sidery, R. Smith, M. Van Der Sluys, A. Vecchio, W. Vousden, and L. Wade, Phys. Rev. D 91, 042003 (2015), arXiv: 1409.7215.

6 B. P. Abbott, et al. (LIGO Scientific Collaboration, Virgo Collaboration), Phys. Rev. Lett. 116, 241102 (2016), arXiv: 1602.03840.

7 B. P. Abbott, et al. (LIGO Scientific Collaboration, Virgo Collaboration), Astrophys. J. 818, L22 (2016), arXiv: 1602.03846.

8 S. Mirshekari, N. Yunes, and C. M. Will, Phys. Rev. D 85, 024041 (2012), arXiv: 1110.2720.

9 N. Yunes, K. Yagi, and F. Pretorius, Phys. Rev. D 94, 084002 (2016), arXiv: 1603.08955.

10 V. A. Kostelecký, and M. Mewes, Phys. Lett. B 757, 510 (2016).

11 C. M. Will, Living Rev. Relativ. 17, 4 (2014), arXiv: 1403.7377.

12 G. Calcagni, Phys. Rev. Lett. 104, 251301 (2010), arXiv: 0912.3142.

13 G. Amelino-Camelia, Nature 418, 34 (2002), arXiv: gr-qc/0207049.

14 P. Hořava, Phys. Rev. D 79, 084008 (2009), arXiv: 0901.3775.

15 A. S. Sefiedgar, K. Nozari, and H. R. Sepangi, Phys. Lett. B 696, 119 (2011), arXiv: 1012.1406.

16 M. Hannam, P. Schmidt, A. Bohé, L. Haegel, S. Husa, F. Ohme, G. Pratten, and M. Pürrer, Phys. Rev. Lett. 113, 151101 (2014), arXiv: 1308.3271 .

17 B. P. Abbott, et al. (LIGO Scientific Collaboration, Virgo Collaboration), Phys. Rev. Lett. 116, 221101 (2016), arXiv: 1602.03841.

18 A. Ghosh, A. Ghosh, N. K. Johnson-McDaniel, C. K. Mishra, P. Ajith, W. Del Pozzo, D. A. Nichols, Y. Chen, A. B. Nielsen, C. P. L. Berry, and L. London, Phys. Rev. D 94, 021101 (2016), arXiv: 1602.02453.

19 A. Ghosh, N. K. Johnson-McDaniel, A. Ghosh, C. K. Mishra, P. Ajith, W. Del Pozzo, C. P. L. Berry, A. B. Nielsen, L. London, LIGOP1700006, ICTS/2017/3 (2017), arXiv: 1704.06784.

20 L. Blanchet, and B. S. Sathyaprakash, Class. Quantum Grav. 11, 2807 (1994).

21 L. Blanchet, T. Damour, and B. R. Iyer, Phys. Rev. D 51, 5360 (1995), arXiv: gr-qc/9501029.

22 K. G. Arun, B. R. Iyer, M. S. S. Qusailah, and B. S. Sathyaprakash, Phys. Rev. D 74, 024006 (2006), arXiv: gr-qc/0604067.

23 C. K. Mishra, K. G. Arun, B. R. Iyer, and B. S. Sathyaprakash, Phys. Rev. D 82, 064010 (2010), arXiv: 1005.0304.

24 N. Yunes, and F. Pretorius, Phys. Rev. D 80, 122003 (2009), arXiv: 0909.3328.

25 T. G. F. Li, W. Del Pozzo, S. Vitale, C. Van Den Broeck, M. Agathos, J. Veitch, K. Grover, T. Sidery, R. Sturani, and A. Vecchio, Phys. Rev. D 85, 082003 (2012), arXiv: 1110.0530.

26 T. Dal Canton, A. H. Nitz, A. P. Lundgren, A. B. Nielsen, D. A. Brown, T. Dent, I. W. Harry, B. Krishnan, A. J. Miller, K. Wette, K. Wiesner, and J. L. Willis, Phys. Rev. D 90, 082004 (2014), arXiv: 1405.6731.

27 S. Klimenko, G. Vedovato, M. Drago, F. Salemi, V. Tiwari, G. A. Prodi, C. Lazzaro, K. Ackley, S. Tiwari, C. F. Da Silva, and G. Mitselmakher, Phys. Rev. D 93, 042004 (2016), arXiv: 1511.05999.

28 S. Klimenko, I. Yakushin, A. Mercer, and G. Mitselmakher, Class. Quantum Grav. 25, 114029 (2008).

29 B. P. Abbott, et al. (LIGO Scientific Collaboration and Virgo Collaboration), Phys. Rev. Lett. 116, 061102 (2016).

30 R. Lynch, S. Vitale, R. Essick, E. Katsavounidis, and F. Robinet, Phys. Rev. D 95, 104046 (2017), arXiv: 1511.05955.

31 N. J. Cornish, and T. B. Littenberg, Class. Quantum Grav. 32, 135012 (2015), arXiv: 1410.3835 .

32 T. B. Littenberg, and N. J. Cornish, Phys. Rev. D 91, 084034 (2015).

33 C. Capano, I. Harry, S. Privitera, and A. Buonanno, Phys. Rev. D 93, 124007 (2016), arXiv: 1602.03509.

34 D. A. Brown, I. Harry, A. Lundgren, and A. H. Nitz, Phys. Rev. D 86, 084017 (2012), arXiv: 1207.6406.

35 I. W. Harry, A. H. Nitz, D. A. Brown, A. P. Lundgren, E. Ochsner, and D. Keppel, Phys. Rev. D 89, 024010 (2014), arXiv: 1307.3562.

36 P. Ajith, N. Fotopoulos, S. Privitera, A. Neunzert, N. Mazumder, and A. J. Weinstein, Phys. Rev. D 89, 084041 (2014), arXiv: 1210.6666.

37 S. Privitera, S. R. P. Mohapatra, P. Ajith, K. Cannon, N. Fotopoulos, M. A. Frei, C. Hanna, A. J. Weinstein, and J. T. Whelan, Phys. Rev. D 89, 024003 (2014), arXiv: 1310.5633.

38 B. P. Abbott, et al. (LIGO Scientific Collaboration, Virgo Collaboration), Phys. Rev. D 93, 122003 (2016), arXiv: 1602.03839.

39 C. Messick, K. Blackburn, P. Brady, P. Brockill, K. Cannon, R. Cariou, S. Caudill, S. J. Chamberlin, J. D. E. Creighton, R. Everett, C. Hanna, D. Keppel, R. N. Lang, T. G. F. Li, D. Meacher, A. Nielsen, C. Pankow, S. Privitera, H. Qi, S. Sachdev, L. Sadeghian, L. Singer, E. G. Thomas, L. Wade, M. Wade, A. Weinstein, and K. Wiesner, Phys. Rev. D 95, 042001 (2017), arXiv: 1604.04324.

40 A. Torres-Forné, A. Marquina, J. A. Font, and J. M. Ibáñez, Phys. Rev. D 94, 124040 (2016).

41 S. A. Usman, A. H. Nitz, I. W. Harry, C. M. Biwer, D. A. Brown, M. Cabero, C. D. Capano, T. D. Canton, T. Dent, S. Fairhurst, M. S. Kehl, D. Keppel, B. Krishnan, A. Lenon, A. Lundgren, A. B. Nielsen, L. P. Pekowsky, H. P. Pfeiffer, P. R. Saulson, M. West, and J. L. Willis, Class. Quantum Grav. 33, 215004 (2016).

42 M. Principe, and I. M. Pinto, Phys. Rev. D 95, 082006 (2017).

43 T. B. Littenberg, J. B. Kanner, N. J. Cornish, and M. Millhouse, Phys. Rev. D 94, 044050 (2016).

44 B. Allen, J. D. E. Creighton, Flanagan, and J. D. Romano, Phys. Rev. D 67, 122002 (2003).

45 B. Allen, J. D. E. Creighton, Flanagan, and J. D. Romano, Phys. Rev. D 65, 122002 (2002).

46 P. J. Green, Biometrika 82, 711 (1995).

47 B. P. Abbott, et al. (LIGO Scientific Collaboration, Virgo Collaboration), Phys. Rev. Lett. 116, 241103 (2016), arXiv: 1606.04855.

48 B. F. Schutz, and M. Tinto, Mon. Not. R. Astron. Soc. 224, 131 (1987).

49 T. A. Apostolatos, C. Cutler, G. J. Sussman, and K. S. Thorne, Phys. Rev. D 49, 6274 (1994).

50 L. Blanchet, Living Rev. Relativ. 17, 2 (2014), arXiv: 1310.1528.

51 T. Damour, Phys. Rev. D 64, 124013 (2001), arXiv: gr-qc/0103018.

52 P. Ajith, M. Hannam, S. Husa, Y. Chen, B. Brügmann, N. Dorband, D. Müller, F. Ohme, D. Pollney, C. Reisswig, L. Santamarla, and J. Seiler, Phys. Rev. Lett. 106, 241101 (2011), arXiv: 0909.2867. 
53 L. Santamarĺa, F. Ohme, P. Ajith, B. Brügmann, N. Dorband, M. Hannam, S. Husa, P. Mösta, D. Pollney, C. Reisswig, E. L. Robinson, J. Seiler, and B. Krishnan, Phys. Rev. D 82, 064016 (2010), arXiv: 1005.3306

54 M. Campanelli, C. O. Lousto, and Y. Zlochower, Phys. Rev. D 74, 041501 (2006), arXiv: gr-qc/0604012.

55 C. Reisswig, S. Husa, L. Rezzolla, E. N. Dorband, D. Pollney, and J. Seiler, Phys. Rev. D 80, 124026 (2009), arXiv: 0907.0462.

56 M. Pürrer, M. Hannam, P. Ajith, and S. Husa, Phys. Rev. D 88, 064007 (2013), arXiv: 1306.2320.

57 M. Pürrer, M. Hannam, and F. Ohme, Phys. Rev. D 93, 084042 (2016), arXiv: 1512.04955.

58 S. Vitale, R. Lynch, V. Raymond, R. Sturani, J. Veitch, and P. Graff, Phys. Rev. D 95, 064053 (2017), arXiv: 1611.01122.

59 C. L. Rodriguez, M. Zevin, C. Pankow, V. Kalogera, and F. A. Rasio,
Astrophys. J. 832, L2 (2016), arXiv: 1609.05916.

60 V. Kalogera, Astrophys. J. 541, 319 (2000), arXiv: astro-ph/9911417.

61 M. Santander, (2016), arXiv: 1606.09335.

62 S. Adrián-Martínez, et al. (Antares Collaboration, IceCube Collaboration, LIGO Scientific Collaboration, and Virgo Collaboration) Phys. Rev. D 93, 122010 (2016), arXiv: 1602.05411.

63 A. Albert, et al. (Antares Collaboration, IceCube Collaboration, LIGO Scientific Collaboration, and Virgo Collaboration) (2017), arXiv: 1703.06298.

64 H. M. Lee, E. O. Le Bigot, Z. H. Du, Z. X. Lin, X. Y. Guo, L. Q. Wen, K. S. Phukon, V. Pandey, S. Bose, X. L. Fan, and M. Hendry, Sci. China-Phys. Mech. Astron. 58, 120403 (2015).

65 X. L. Fan, Sci. China-Phys. Mech. Astron. 59, 640001 (2016).

66 Z. J. Cao, Sci. China-Phys. Mech. Astron. 59, 110431 (2016). 